MRI-PHY $/ 21 / 95$

TIFR-TH/95-52

hep-th/9510233

\title{
Notes on a singular Landau-Ginzburg family
}

\author{
Debashis Ghoshall \\ Tata Institute of Fundamental Research \\ Homi Bhabha Road, Bombay 400 005, India \\ $\&$ \\ Mehta Research Institute of Mathematics 8 Mathematical Physics \\ 10 Kasturba Gandhi Road, Allahabad 211 002, India
}

\begin{abstract}
We study some properties of a singular Landau-Ginzburg family characterized by the multi-variable superpotential $W=-X^{-1}\left(Y_{1} Y_{2}\right)^{n-1}+\frac{1}{n}\left(Y_{1} Y_{2}\right)^{n}-Y_{3} Y_{4}$. We will argue that (the infra-red limit of) this theory describes the topological degrees of freedom of the $c=1$ string compactified at $n$ times the self-dual radius. We also briefly comment on the possible realization of these line singularities as singularities of Calabi-Yau manifolds.
\end{abstract}

October 1995

1 E-mail: ghoshal@theory.tifr.res.in. 


\section{Introduction}

Non-critical string theories prove to be instrumental in our understanding of some of the fundamental issues in highly simplified situations (see [1] for a review and reference to the original literature). Symmetries, behaviour of the perturbation series to all orders in string coupling, nature of non-perturbative effects, etc. are questions that these 'toy' models have addressed with some success. The simplicity of these theories derive from the fact that they are conventionally thought of as strings moving in $d \leq 2$ dimensions, where only the topological (quantum mechanical) degrees of freedom survive. The most interesting of these is $d=2$ : a free scalar field of central charge one on a circle of radius $R$, coupled to gravity - the so called $c=1$ string. This has a propagating massless degree of freedom with momentum quantized in units of $1 / R$.

The topological properties of non-critical string theories are best described in a formalism that makes them manifest. This is the subject of topological gravity [2] and topological string theory, which for $d<2$ is reviewed in [3]. The same story for $c=1$ string is more complex. The most symmetric case of $c=1$ string at the radius $R=1$, (self-dual value under $R \rightarrow 1 / R$ duality), variously admits description as double-scaled Penner model [4], twisted $S L(2, \mathbf{R})_{3} / U(1)$ coset model[5], topological Landau-Ginzburg model[6] [7] [8] and its geometrization as a non-compact Calabi-Yau space [9] and Kontsevich-Penner type matrix model[10] (see also [11]). All these highlight different aspects of this theory and testify to its rich structure.

Topological string theories also arise from critical strings with $N=2$ supersymmetry, and are useful in studying a class of physical operators, the chiral ring, in a simplified scenario which makes the other operators of the theory BRS-exact [12]. Many of the interesting physical quantities are tractable in this framework 13 114 and using mirror symmetry 15, leads to some spectacular exact results for string compactification on Calabi-Yau threefolds.

The topological theories of 'toy' non-critical and more physical critical strings turn out to be intimately related. In [16] it was shown that the 'Calabi-Yau phase' of the $c=1$ string at the self-dual radius captures the universal local geometry (near the nodal point to be) of a generic Calabi-Yau threefold as it degenerates into a conifold[177]. A consequence of this is the fact that the topological free energy of the B-twisted sigma model[12] on the Calabi-Yau space has a universal singular part (as a function of the modulus related to the conifold). Furthermore, from $c=1$ string theory, this is given by the genus expansion 
of its free energy! This in turn determines some of the couplings of the effective field theory of the type II string and provides another evidence 18 in favour of the remarkable non-perturbative duality between type II and heterotic strings [19].

In [20], the physical singularity of the low energy effective field theory of a string

near a conifold was traced to the infra-red singularity due to the emergence of a new light (solitonic) black hole degree of freedom. This leads to the tantalizing possibility of a dynamical non-perturbative process where the topology of the compactification changes while the underlying string theory remains smooth[21]. This suggests that one should study other singularities of Calabi-Yau spaces, and as in the case of the simple conifold singularity, non-critical string theories might help us understand some of the universal properties of such degenerations.

With this motivation we will propose and study a class of topological Landau-Ginzburg theories. They are characterized by singular superpotentials. In Sec.2, we will introduce the models and argue that they describe the topological degrees of freedom associated with the $c=1$ string at $n$ times the self-dual radius. In Sec.3, we will identify the 'tachyons' in terms of the Landau-Ginzburg variables and find the selection rule for correlators, which are then computed in Secs.4 and 5. We find that while the tree-level correlators agree with the matrix model results, there is a discrepancy in the genus- 1 correlation functions. We will argue that this is because the Landau-Ginzburg theory describes only the topological sector which does not include the intermediate tachyons with fractional momenta. In Sec.6, we discuss their geometry and find non-compact Calabi-Yau spaces and their singularities. Finally, in Sec.7, we will briefly comment on their realization as singularities of compact Calabi-Yau threefolds.

\section{The singular family of superpotentials}

Let us first recall the Landau-Ginzburg model for the $c=1$ string at the self-dual radius [6] [7] [8]. The singular superpotential $W(X)=-\mu X^{-1}$ is a characteristic feature of this theory and determines the dynamics of the 'tachyon' degrees of freedom completely. The Landau-Ginzburg theory manifestly has the topological symmetry algebra with the topological central charge $\hat{c}=3$. This value of $\hat{c}$ is critical in the sense that the free energy of such theories is naturally well-defined at all genus without any operator insertion[2]. Remarkably $\hat{c}$ is also 3 for topological models based on Calabi-Yau spaces, reflecting the 
fact that their (complex) dimension is 3. Given the close connection between LandauGinzburg and Calabi-Yau models [22], it is natural to expect that the $c=1$ string also has a 'Calabi-Yau phase'. Indeed one finds a non-compact analog of Calabi-Yau manifolds[9] defined by the zero locus of the (augmented) superpotential

$$
W=-\mu x^{-1}+y_{1} y_{2}-y_{3} y_{4}
$$

in the non-compact weighted projective space $\mathbf{P}_{-2,1,1,1,1}^{4}$. The additional quadratic terms do not change the value of the central charge and only make up for the missing coordinates. Nor do they affect any result obtained for the tachyon correlators using the superpotential $1 / X$.

The non-compact Calabi-Yau space given by the eq.(2.1) describes the universal local geometry (near the would be nodal singular point) of a generic (compact) Calabi-Yau threefold as it degenerates into a conifold[16]. This is apparent in the coordinate patch $(x \neq 0)$ where we find

$$
y_{1} y_{2}-y_{3} y_{4}=\mu
$$

In the above cosmological constant $\mu$ of the $c=1$ string is the complex structure modulus that tunes us to the conifold singularity at $\mu=0$.

The conifold locus (2.2) also has a well known interpretation in the $c=1$ string theory. The string BRS cohomology of these theories has an infinite number of nonstandard physical operators of zero ghost number 23]. By virtue of being in the BRS cohomology they also have dimension zero. The product of such operators is therefore free of singularity and forms a polynomial ring, called the ground ring[24]. This ring and the associated variety govern the theory and its symmetries. Conifold (2.2) is the complexification of this ground variety [16].

The ground ring and symmetries of $c=1$ string have been studied as a function of the radius of compactification, and more generally at all points in the moduli space of the $c=1$ conformal field theory [25]. At a set of special points - called the ADE points in the CFT moduli space, they are related to the Kleinian singularities [26] [27]. In this paper, we will only be concerned with the $A_{n}$-type singularities which describe the $c=1$ theory compactified on $n$ times the self-dual radius ( $R=n$ in suitable units). For them the locus of the ground variety bears a close resemblance to eq.(2.2). However, we will see that there are some essential differences between $R=1$ and $R=n>1$. 
Recall that the (unperturbed) ground ring describes the singular variety

$$
\frac{1}{n}\left(y_{1} y_{2}\right)^{n}-y_{3} y_{4}=0
$$

We will assume that this is related to a multi-variable superpotential just as eq.(2.2) is to (2.1). Unlike the theory at the self-dual radius, the $y$-part is not quadratic and hence contributes to the central charge. It is easy to work out the $\mathrm{U}(1)$ charge assignment for the Landau-Ginzburg fields corresponding to the coordinates $y$ 's such that the superpotential has charge unity. Let $q_{i}$ denote the $\mathrm{U}(1)$ charge of the field $Y_{i}$, then

$$
q_{1}=q_{2}=\frac{1}{2 n} \quad q_{3}=q_{4}=\frac{1}{2}
$$

The contribution to the topological central charge of these fields is therefore $\hat{c}_{Y}=\sum(1-$ $\left.2 q_{i}\right)=2(n-1) / n$. The total topological central charge of the $c=1$ string is independent of the radius of compactification 28] and hence is $\hat{c}=3$ for all integer $n$. The deficit therefore has to be made up by an additional superfield $X$ with

$$
q_{X}=-1 / n
$$

that contributes $\hat{c}_{X}=(n+2) / n$ to the central charge.

This brings us to a singular Landau-Ginzburg family characterized by the superpotential

$$
W=-\mu X^{-1}\left(Y_{1} Y_{2}\right)^{n-1}+\frac{1}{n}\left(Y_{1} Y_{2}\right)^{n}-Y_{3} Y_{4}
$$

generalizing the $n=1$ case given in eq.(2.2)above. This is of course not the most general possible form as one can add terms of the form $t_{l} X^{-l}\left(Y_{1} Y_{2}\right)^{n-l},(l=2, \cdots, n)$, and even terms where the powers of $Y_{1}$ and $Y_{2}$ differ 2 . All of these are compatible with the $\mathrm{U}(1)$ charge assignment, and polynomial in the $Y$ fields and moreover are related closely to the mini-versal deformation of the singular ground variety (2.3) [29]. However, in the first non-trivial case $(n=2)$ at least, explicit computation shows that the extra term $t X^{-2}$ makes no difference in the computation of the correlators - the final result are the same with or without this term. Although we have no general proof for the above empirical observation, we will, for simplicity, work with the superpotential (2.6). The general form will be useful in the later sections when we discuss the singularities and their possible realization in compact Calabi-Yau threefolds.

2 Terms where $Y_{1}$ and $Y_{2}$ have different powers are related to those where they the same by the $w_{\infty}$ symmetry in the case of the $c=1$ string [29]. 


\section{Landau-Ginzburg operators and selection rule for correlators}

The standard procedure of determining the BRS invariant physical operators (chiral ring) of a topological Landau-Ginzburg theory via the Jacobian ideal does not work with a singular superpotential. For the theory with $W=1 / X$, all positive and negative powers of the superfield $X^{k-1}, k \in \mathbf{Z}$ were proposed to be the physical states[6]. These were identified to the 'tachyons' of momenta $k$ of the $c=1$ string theory. Recall that at the self-dual radius of compactification all momenta are quantized to be integer valued.

For the theory defined by the superpotential (2.6), let us consider the following physical operators

$$
T_{k}=X^{k-1}\left(Y_{1} Y_{2}\right)^{n-1}, \quad k \in \mathbf{Z}
$$

as a simple generalization of the above idea. The genus- $g$ correlation function of $N$ such operators $\left\langle T_{k_{1}} \cdots T_{k_{N}}\right\rangle$ could be non-zero only if the U(1) charge conservation

$$
\sum_{i=1}^{N}\left(q\left(T_{k_{i}}\right)-1\right)=(g-1)(\hat{c}-3)
$$

law holds. For the critical topological theory we are considering $\hat{c}=3$, and the RHS of the above vanishes. Substituting for the U(1) charge $q$, we find the conservation law

$$
\sum_{i=1}^{N} k_{i}=0
$$

independent of genus $g$, exactly as in the theory at the self-dual radius.

This suggests that the operators (3.1) are the tachyons of the $c=1$ string at radius $R=n$. However, the allowed values of momenta at this radius are in units of $1 / n$. The operators $T_{k}$ in eq. (3.1) cannot therefore account for all the tachyons of the theory. They describe only a subset of tachyons, namely those with integer momenta. Interestingly, this subset is special in the sense that additional discrete states appear precisely at these momenta. The rest of the tachyons are in the 'twisted sector'. They do not have any discrete state associated with them 25].

The collection of integer momentum tachyons and the associated discrete states are remnants of the higher modes of the string[1], and form a topological sector of the $c=1$ string at radius $R=n$. We conjecture that only these topological degrees of freedom are described by the Landau-Ginzburg theory with the superpotential (2.6). 
The physical states (3.1) of course do not exhaust the admissible physical states of the Landau-Ginzburg theory. In general one can introduce operators of the form

$$
\mathcal{O}_{p ; r, s}=X^{p-1} Y_{1}^{r+n-1} Y_{2}^{s+n-1}
$$

labelled by three integers $p \in \mathbf{Z}, r, s>-n$. The selection rule for $N$ such states is

$$
\sum_{i=1}^{N}\left(p_{i}-\frac{r_{i}+s_{i}}{2}\right)=0
$$

The operator $\mathcal{O}$ can therefore be thought of as carrying momentum $k=p-\frac{r+s}{2}$. It will be interesting to investigate if these are related to the discrete states of the $c=1$ string.

\section{Residue, contact term and genus-0 correlators}

The simplest correlation function to evaluate is the three-point function on the sphere. The worldsheet has no modulus and the three-point function is simply given by the residue formula in the Landau-Ginzburg theory [30] [31]:

$$
\begin{aligned}
\left\langle T_{k_{1}} T_{k_{2}} T_{k_{3}}\right\rangle_{g=0} & \equiv \operatorname{Res}_{W}\left(T_{k_{1}} T_{k_{2}} T_{k_{3}}\right) \\
& =\operatorname{Res}_{W}\left[\frac{T_{k_{1}} T_{k_{2}} T_{k_{3}}}{\prod \partial W / \partial x_{j}}\right] \\
& =\operatorname{Res}_{W}\left[\frac{x^{k_{1}+k_{2}+k_{3}+1} y_{1} y_{2}}{\left(x y_{1} y_{2}-(n-1)\right)^{2} y_{3} y_{4}}\right] \\
& =\operatorname{Res}_{W}\left[\frac{x y_{1} y_{2}}{\left(x y_{1} y_{2}-(n-1)\right)^{2} y_{3} y_{4}}\right] \delta_{k_{1}+k_{2}+k_{3}, 0}
\end{aligned}
$$

where in the last step we have used the momentun conservation condition (3.2).

The multivariable residue is discussed in detail in [32]. Intutively it is the coefficient of the simple 'pole' in the right variables. To this end, we notice that after imposing the momentum conservation condition (3.2), the variables $x, y_{1}$ and $y_{2}$ always appear in the combination $x y_{1} y_{2}$. Defining new variables $z_{1}=x y_{1} y_{2}$ and $z_{i+1}=y_{i}$ for $i=1, \cdots, 4$; we find that there are poles at $z_{1}=(n-1)$ and $z_{2}=z_{3}=z_{4}=z_{5}=0$. Each of these poles

is along a (complex) codimension one hypersurface of $\mathbf{C}^{5}$, which can be encircled by a one (real) dimensional contour. The direct product of these contours enclose the critical points where all the poles intersect. With this prescription the residue in the last step of eq.(4.1) is unity. The three-point function of the tachyons is the momentum conserving delta function giving the expected answer. 
Now we come to first non-trivial case, namely the four-point function on the sphere. The contribution from the bulk of the moduli space is given by

$$
\begin{aligned}
\left.\left\langle T_{k_{1}} T_{k_{2}} T_{k_{3}} T_{k_{4}}\right\rangle_{g=0}\right|_{\text {bulk }} & =\left.\frac{\partial}{\partial t_{k_{4}}} \operatorname{Res}_{W+t_{k_{4}} T_{k_{4}}}\left(T_{k_{1}} T_{k_{2}} T_{k_{3}}\right)\right|_{t_{k_{4}}=0} \\
& =\left(1-k_{4}\right) \delta_{\sum k, 0}
\end{aligned}
$$

as one would expect from a topological theory of Landau-Ginzburg matter 31]. But this is a (topological) string theory, and involves integration over the one dimensional moduli space of a sphere with four punctures. Therefore, in addition to the above, there are corrections from the boundaries of the moduli space. This happens when the location of $T_{k_{4}}$ 'collide' with the other tachyon insertions, which are fixed by the Killing symmetries of the sphere. The contribution from the boundaries can be neatly encoded by defining contact terms between tachyons [33].

A suitable choice of contact term turns out to be

$$
C_{W}\left(T_{k_{1}}, T_{k_{j}}\right)=\sum_{j=1}^{5} \frac{\partial}{\partial x_{j}}\left(\frac{T_{k_{1}} T_{k_{2}}}{\partial W / \partial x_{j}}\right)_{\operatorname{sign} q_{j}}
$$

The subscript $\left\{\operatorname{sign} q_{j}\right\}=\{-++++\}$ is the mnemonic that in the first term, after differentiating wrt $x$, we only keep terms with negative powers of $x$, and similarly the $j$ th term is retained only if $y_{j}$ appears with positive power. The contact term thus defined is a generalization of that in [6] and is easily evaluated to be

$$
\begin{aligned}
C_{W}\left(T_{k_{1}}, T_{k_{2}}\right)=( & \left(k_{1}+k_{2}\right) T_{k_{1}+k_{2}} \theta\left(-k_{1}-k_{2}\right) \\
& \left.+2 n \frac{x^{k_{1}+k_{2}-1}\left(y_{1} y_{2}\right)^{n-1}}{\left(x y_{1} y_{2}-(n-1)\right)}-2 \frac{x^{k_{1}+k_{2}}\left(y_{1} y_{2}\right)^{n}}{\left(x y_{1} y_{2}-(n-1)\right)^{2}}\right)
\end{aligned}
$$

The first term in the RHS above is a tachyon and is exactly the same as in the $n=1$ case for which the second and third term cancel out. We will see below that only this term contributes to the tachyon correlators.

Using the contact term (4.4), the boundary contribution when $T_{k_{4}}$ collides with, say, $T_{k_{3}}$ is

$$
\left\langle T_{k_{1}} T_{k_{2}} C_{W}\left(T_{k_{3}}, T_{k_{4}}\right)\right\rangle=\left(k_{3}+k_{4}\right) \theta\left(-k_{3}-k_{4}\right) \delta_{\sum k, 0}
$$

The non-tachyonic terms in (4.4) have higher poles and do not contribute. Adding the bulk and boundary contributions, we get the correlation function of four tachyons on the sphere

$$
\left\langle T_{k_{1}} T_{k_{2}} T_{k_{3}} T_{k_{4}}\right\rangle_{g=0}=(1-\max |k|) \delta_{\sum k, 0}
$$


This result agrees with the matrix model answer [34] apart from an overall normalization factor of $R=n$. Restricting to the kinematical configuration where $k_{j}>0$ for $j \geq 4$, all genus-0 correlators agree with the matrix model result. A general proof of this can be worked out along the lines of [6]. In other kinematical configurations, the answers agree in all cases that we have checked.

Two comments are in order here. Firstly, although the genus-0 correlators are insensitive to the radius of compactification, the fact that the Landau-Ginzburg model motivated from the ground ring of $c=1$ string reproduces them correctly provides a non-trivial check. Secondly, by taking the momenta $k$ to be valued formally in $\mathbf{Z} / n$, one gets the genus-0 correlators of all tachyons. However, the fields $T_{k}$ with fractional $k$ do not, strictly speaking, belong to the physical states of the topological theory.

\section{Correlation functions on the torus}

We now come to the correlation functions on higher genus Riemann surfaces. Unfortunately these are difficult to compute in string theory even for the simple topological Landau-Ginzburg models.

The situation is perhaps best understood for topological Landau-Ginzburg theory with superpotential $W=1 / X[8]$. Tachyons with positive momenta are primaries and those with negative momenta are to be thought of as gravitational descendants. More precisely, a tachyon with negative momentum is thought of a linear combination of different terms each of which is a product of gravitational and 'matter' degrees of freedom:

$$
T_{-k}=\sum_{i=0}^{k} \prod_{j=1}^{i}(j-k) \sigma_{i} T_{-k+i}
$$

For a given Riemann surface, the conservation law uniquely picks up one of these terms so that the gravitational part $\sigma_{i}$ accounts for the dimension of the relevant moduli space. This is the 'picture changing' hypothesis for the tachyons [8].

In the appropriate 'picture' the correlator factorizes into purely gravitational and matter contributions. The former is given by topological gravity[2]. The matter part is more complicated. But in each genus, the contribution from the bulk of the moduli space is given by the handle operator insertion prescription for topological Landau-Ginzburg matter[31]. The various boundary contributions then come from contact terms between 
handles. For the $c=1$ string at the self-dual radius these combine to give the $W_{\infty}$ constraints 8 .

As an example consider the one-point function of $T_{-k}$ on the torus. The moduli space is one dimensional, so the correct insertion of the tachyon is in the '1-picture'

$$
\begin{aligned}
\left\langle T_{-k}\right\rangle_{g=1} & =(1-k)\left\langle\sigma_{1}\right\rangle_{g=1}\left\langle T_{-k+1}\right\rangle_{g=1} \\
& =\frac{(1-k)}{24} \operatorname{Res}_{W}\left[\frac{\partial^{2} W / \partial x^{2}}{\partial W / \partial x} T_{-k+1}\right]
\end{aligned}
$$

In the above we have used $\left\langle\sigma_{1}\right\rangle_{g=1}=1 / 24$ in topological gravity [2] and that the handle operator is $\partial^{2} W / \partial x^{2}$ [31]. All $1 \rightarrow N$ correlators on the torus, that is correlators involving one negative and $N$ postive momentum tachyons can be obtained from (5.2) by starting with the perturbed superpotential $W+\sum t_{k} T_{k}$, and differentiating it $N$ times in the appropriate couplings $t_{k}$ 's. In this case, it turns out that (5.2) gives the complete answer and that there is no contribution from the boundary [8].

We will assume that the above expression (with appropriate modification to take into account several variables), is true in the Landau-Ginzburg theory with superpotential (2.6). To evaluate the correlator, we need the '1-picture' for the tachyon $T_{-k}$. The proposal we make for this is the following:

$$
T_{-k} \sim(1-k) \sigma_{1} \frac{T_{-k+1}}{T_{1}}=(1-k) \sigma_{1} x^{-k}
$$

The appearance of the puncture operator $T_{1}=\left(y_{1} y_{2}\right)^{n-1}$ in the above is necessitated by the $\mathrm{U}(1)$ charge conservation. An analogous situation is the closely related formula

$$
C_{W}\left(\sigma_{s} \cdot \phi_{i}, P\right) \sim \sigma_{s-1} \cdot \phi_{i}
$$

of [35] for the contact term between a gravitational descendant and the puncture operator in Landau-Ginzburg matter coupled to gravity. In the theory with $W=1 / X$, the puncture operator is identity and does not appear explicitly.

The proposed form of the tachyon in '1-picture' can be checked by evaluating the genus-0 four point function of three positive and one negative momentum tachyons:

$$
\left\langle T_{k_{1}} T_{k_{2}} T_{k_{3}} T_{-k_{4}}\right\rangle_{g=0}=\left\langle\sigma_{1}\right\rangle_{g=1} \operatorname{Res}_{W}\left(T_{k_{1}} T_{k_{2}} T_{k_{3}} T_{-k_{4}+1}\right)=\left(1-k_{4}\right) \delta_{\sum k, 0}
$$

where, $k_{i} \geq 0$ for all $i=1, \cdots, 4$. 
Now we will calculate the one-point function of $T_{-k}$ on the torus. The handle operator is given by the determinant of the matrix of the second derivatives of the superpotential [31]. Using picture changing, we have

$$
\left\langle T_{-k}\right\rangle_{g=1}=(1-k)\left\langle\sigma_{1}\right\rangle_{g=1} \operatorname{Res}_{W}\left[\frac{\operatorname{det}\left\|\partial_{i} \partial_{j} W\right\|}{\prod \partial_{i} W} \frac{T_{-k+1}}{T_{1}}\right]
$$

Substituting the explicit form of the handle operator

$$
\operatorname{det}\left\|\partial_{i} \partial_{j} W\right\|=-2 x^{-5}\left(y_{1} y_{2}\right)^{3 n-5}\left(x y_{1} y_{2}-(n-1)\right)\left((2 n-1) x y_{1} y_{2}-(n-1)(n-2)\right)
$$

we find that

$$
\left\langle T_{-k}\right\rangle_{g=1}=-\frac{(2 n-1)}{12}
$$

This answer unfortunately does not agree with the one obtained from matrix model (except for $n=1$ ), which at $R=n$ is

$$
\left\langle T_{-k}\right\rangle_{g=1}^{M M}=-\frac{1}{24}\left(n+\frac{1}{n}\right)
$$

The result of the Landau-Ginzburg model is an integral multiple of that in the theory at the self-dual radius. In the case of $n=2$, it is easy to check that the result is the same even with the superpotential $W=t X^{-2}-\mu X^{-1} Y_{1} Y_{2}+\frac{1}{2}\left(Y_{1} Y_{2}\right)^{2}-Y_{3} Y_{4}$, which retains all terms related to the deformation of the ground ring $(2.3)$, and this is likely to be the case for all $n$.

Likewise we find the torus two-point function. Start with the perturbed superpotential and differentiate (5.5) to get

$$
\left\langle T_{-k} T_{k}\right\rangle_{g=1}=\frac{(2 n-1)}{24}(1-k)\left(k^{2}-k-2\right)
$$

Although the functions involved and their individual contribution to the residue are different from (5.5), the result, once again, is the same integral multiple of that at the self-dual radius. While the matrix model result

$$
\left\langle T_{-k} T_{k}\right\rangle_{g=1}^{M M}=\frac{1}{24}(1-k)\left(n k^{2}-n k-\left(n+\frac{1}{n}\right)\right)
$$

again disagrees with (5.9).

The hypothesis that the Landau-Ginzburg theory describes only the topological degrees of freedom of the $c=1$ string at the radius $R=n$, could provide a resolution to the descrepancy. If so, it is not surprising that we do not obtain the expected answer in this formalism. To match with matrix model, one needs to take into account the effect of the intermediate tachyons (of fractional momenta) in the twisted sector. It is interesting that the difference shows up for the first time in loop computations where all states contribute. 


\section{Calabi-Yau phase and singularities}

Landau-Ginzburg models are closely associated to Calabi-Yau spaces [22] of (complex) dimension $\operatorname{dim}_{\mathbf{C}}=\hat{c}$ (for integer values of the central charge). The zero locus of the superpotential defines the manifold as an hypersurface in some appropriate projective space. Witten has argued that the two descriptions can be thought of as different phases of the same theory [22].

The Calabi-Yau phase of the $c=1$ string theory at the self-dual radius [9] is particularly interesting. It describes the universal local geometry of a generic Calabi-Yau threefold as it degenerates into a simple conifold singularity [16]. A conifold is Calabi-Yau threefold with an isolated singular point of zero multiplicity [17. Such canonical singularities are generically present in the (complex structure) moduli space of threefolds and moreover are only a finite distance away from a typical point there. It turns out that near the isolated nodal point on the degenerate manifold, it assumes the universal form (2.2) with $\mu=0$. Small non-zero value of $\mu$ corresponds to the nearly degenerate situation. Thus one identifies the cosmological constant $\mu$ of the $c=1$ string with the complex modulus that tunes to the conifold. The Calabi-Yau space related to the $c=1$ string at the self-dual radius is given by the hypersurface defined by the zero of the superpotential (2.1). This equation is quasi-homogeneous with the weights (2.4)(2.5) (with $n=1$ ), and is therefore an equation in the weighted projective space $\mathbf{P}_{-2,1,1,1,1}^{4}$. Notice that this as well as the Calabi-Yau space, which is exactly the conifold, are non-compact.

Figuratively speaking, the Calabi-Yau phase of $c=1$ string at the self-dual radius is an approximation to a threefold near a conifold point as the harmonic oscillator potential $x^{2}$ is to a generic potential at a simple critical point. A consequence of this approximation of local geomtery by the $c=1$ string is the fact that the universal physics dominated by the formation of the singularity is described by the non-critical string theory. In particular, the singular part of the topological free energy of a B-twisted[12] sigma model based on any degenerating Calabi-Yau manifold is given by the free energy of the $c=1$ string as a function of $\mu[16]$. This has an exact expression involving the virtual Euler number of the moduli space of genus- $g$ Riemann surfaces [36] [4] [5]. Geometrically this free energy is related to a certain period of the holomorphic 3-form of the manifold[17]. Physically they determine, for type II strings, some $F$-terms in the effective field theory [13] [14]. 
Since the Calabi-Yau phase of the $c=1$ string at the self-dual radius has a useful realization, it is natural to study the same for the theory at $R=n$ defined by the superpotential (2.6). Repeating similar argument, it follows that the Calabi-Yau space is defined by the zero locus of the superpotential 3

$$
W=\frac{1}{n}\left(y_{1} y_{2}\right)^{n}-y_{3} y_{4}-\mu x^{-1}\left(y_{1} y_{2}\right)^{n-1}+\sum_{l=2}^{n} t_{l} x^{-l}\left(y_{1} y_{2}\right)^{n-l}
$$

Due to the weight assigments (2.4) and (2.5) of the fields, $W=0$ defines a hypersurface in the weighted projective space $\mathbf{P}_{-2,1,1, n, n}^{4}$. This ambient projective space is again noncompact and also has singularities characteristic of weighted projective spaces. Similary the hypersurface $W=0$ is non-compact.

In the affine patch $(x \neq 0)$, the Calabi-Yau manifold in question is given by the (polymonial equation)

$$
\frac{1}{n}\left(y_{1} y_{2}\right)^{n}-y_{3} y_{4}-\mu\left(y_{1} y_{2}\right)^{n-1}+\sum_{l=2}^{n} t_{l}\left(y_{1} y_{2}\right)^{n-l}=0
$$

in $\mathbf{C}^{4}$. For generic values of the parameters $\mu$ and $t_{l}$ the hypersurface (6.2) is non-singular. However, it develops singularity along codimension one subspace of the parameter space $\left(\mu, t_{l}\right)$. These in turn intersect among themselves along higher codimension subspaces. The origin of this parameter space defines the most singular manifold (2.3).

There is an important difference in the case $n>1$ compared to $n=1$. The singularity here is not isolated. The hypersurface (2.3) is singular along a (complex) 1-dimensional subspace. The locus of singularity is given by

$$
\left(y_{1} y_{2}\right)^{n-1}=0 \quad y_{3}=y_{4}=0
$$

which is a pair of intersecting lines in $\mathbf{C}^{2}$ defined by $y_{1}$ and $y_{2}$. This is a two-sided real 2-dimensional cone with circular base. This singularity is $(n-1)$-fold degenerate.

As an illustration consider the simplest case, $n=2$. The moduli space of the singularity is two dimensional with coordinates $\mu$ and $t_{2}$. The most singular case is for $\mu=t_{2}=0$, for which the manifold is singular along $y_{1} y_{2}=0$. For the codimension one locus $t_{2}=\mu^{2} / 2$, the line singularity persists, but they are along $y_{1} y_{2}=\mu$ in the $y_{1} y_{2}$ plane, and is still

3 Here we use the general form of the superpotential. 
one-fold degenerate. The real geometry of this is that of a hyperboloid of revolution. For $t_{2}=0$, but $\mu \neq 0$, there is only an isolated singularity at the origin.

The locus of singularity $(6.3)$ is also familiar from the $c=1$ string. It is merely the complexification of the singularity of the ground variety [25], which continues to remain singular under a perturbation by the cosmological operator 29]. In $c=1$ string, the singular locus is identified with the fermi level of the matrix model free fermions [24] [25]. In the latter description, tachyons are thought of as small deformations (ripples) on the fermi surface[1]. In the topological description, it is only the intermediate tachyons with fractional momenta that are localized degrees of freedom on the singular locus 25] [29]. Notice that the multiplicity of degeneracy of the singularity equals the types of intermediate tachyons in the twisted sector (tachyons with the same fractional part of momentum). In the limit $R \rightarrow \infty$, the singular locus becomes infinitely degenerate corresponding to the fact that the intermediate tachyons form a continuum there. The two-dimensional surface of singularity dominates the dynamics, and should perhaps be identified with the conventional target space.

The two sides of the fermi sea are connected when we identify it to the complex singularity. On the other hand, from matrix model, it has recently been suggested that both sides of the fermi sea should be taken into account for a consistent string interpretation [37]. This brings in states that correspond to the negatively dressed ('Seiberg disallowed' [1]) discrete states of the Liouville theory. Whereas from the ground ring of $c=1$ string, these precisely give rise to the deformations considered above 29.

\section{Realization of the singularities in compact Calabi-Yau spaces}

We will now briefly discuss the possibility of realizing the singularities described above in compact Calabi-Yau spaces. Indeed these singularities do occur in compact threefolds, and it is rather easy to find such examples. The first one we will cite is the ubiquitous quintic. Consider the hypersurface

$$
\left(y_{1} y_{2}\right)^{2} y_{5}-\left(y_{3} y_{4}-\mu y_{1} y_{2}\right) y_{5}^{3}+t_{2} y_{5}^{5}=0
$$

in $\mathbf{P}^{4}$. This has the $A_{2}$ type singularity in the coordinate patch $\left(y_{5} \neq 0\right)$. Similarly the degree 8 hypersurface

$$
\frac{1}{3}\left(y_{1} y_{2}\right)^{3} y_{5}-\left(y_{3} y_{4}-\mu\left(y_{1} y_{2}\right)^{2}\right) y_{5}^{2}+t_{2} y_{1} y_{2} y_{5}^{3}+t_{3} y_{5}^{4}
$$


$=0$ in $\mathbf{P}_{1,1,2,2,2}^{4}$ has the $A_{3}$ singularity. Other examples like $\mathbf{P}_{1,2,3,6,6}^{4}[18]$ ( $A_{5}$ type) and $\mathbf{P}_{1,1,1,6,9}^{4}[18]$ ( $A_{8}$ type) can be found by searching among known Calabi-Yau spaces.

These are canonical singularities, and are therefore only a finite distance away from a generic smooth point of the moduli space [38]. The effective field theory will again reflect the singularity of the metric on the moduli space, and it will be interesting to see if these can be resolved in a way analogous to the picture of [20].

The topological one-loop free energy that we have found for the $A_{n}$ singularity using the singular Landau-Ginzburg model is $F_{1}=-\frac{2 n-1}{12} \log \mu$. It will be interesting to check if these numbers are related to the degenerations of compact Calabi-Yau. One should remember that this may not be full answer, as it does not agree with the matrix model result. A related point is that the singular locus $y_{1} y_{2}=\mu$ could be part of a curve of non-zero genus and have a non-zero fundamental group, and it was found that the chiral ring analysis is not sufficient in such cases 39].

Finally, the Landau-Ginzburg method discussed here can be extended to the case of $D_{n}, E_{6}, E_{7}$ and $E_{8}$ type singularities by appealing to the corresponding ground ring of $c=1$ string theory[25]. We hope that the $A_{n}$ models described here and their extensions will be helpful in understanding different singularities of Calabi-Yau spaces.

Acknowledgement: I am grateful to Camillo Imbimbo, Kirti Joshi, Samir Mathur, Sunil Mukhi, Kapil Paranjape, Ashoke Sen and Cumrun Vafa for valuable discussion. Part of this work was done at the Tata Institute of Fundamental Research. I would like to thank the Theoretical Physics Group there for hospitality.

Note added: After completing this note, a recent preprint [40] appeared that analyzes the $A_{n}$ singularities in detail and relates them to the singularities of $K 3 \times T^{2}$. 


\section{References}

[1] I. Klebanov, Lectures at the ICTP Spring School (1991), hep-th/9108019;

P. Ginsparg and G. Moore, Lectures at the TASI Summer School (1992), hepth/9304011.

[2] E. Witten, Nucl. Phys. B340 (1990) 281.

[3] R. Dijkgraaf, Lectures at NATO ASI, Cargèse (1991), Eds. J. Fröhlich et al, Plenum Press, hep-th/9201003.

[4] J. Distlerand C. Vafa, Mod. Phys. Lett. A6 (1991) 259.

[5] S. Mukhi and C. Vafa, Nucl. Phys. 407 (1993) 667.

[6] D. Ghoshal and S. Mukhi, Nucl. Phys. B425 (1994) 173.

[7] A. Hanany, Y. Oz and R. Plesser, Nucl. Phys. B425 (1994) 150.

[8] D. Ghoshal, C. Imbimbo and S. Mukhi, Nucl. Phys. B440 (1995) 355.

[9] D. Ghoshal and S. Mukhi, in the Proceedings of the International Colloquium on Modern Quuantum Field Theory, Bombay (1994), available at the home page of the Theoretical Physics Group of the Tata Institute.

[10] C. Imbimbo and S. Mukhi, Nucl. Phys. B449 (1995) 553.

[11] R. Dijkgraaf, G. Moore and R. Plesser, Nucl. Phys. B394 (1993) 356.

[12] E. Witten, in Essays on Mirror Manifold, Ed. S.T. Yau, International Press.

[13] M. Bershadsky, S. Cecotti, H. Ooguri and C. Vafa, Nucl. Phys. B405 (1993) 279; Comm. Math. Phys. 165 (1994) 311.

[14] I. Antoniadis, E. Gava, K. Narain and T. Taylor, Nucl. Phys. B413 (1994) 162.

[15] Essays on Mirror Manifolds, Ed. S.T. Yau, International Press.

[16] D. Ghoshal and C. Vafa, Nucl. Phys. B453 (1995) 121, hep-th/9506122.

[17] P. Candelas, A.M. Dale, C.A. Lütken, and R. Schimmrigk, Nucl. Phys.B298 (1988) 493; P.S. Green and T. Hübsch, Phys. Rev. Lett. 61 (1988) 1163; Comm. Math. Phys.119 (1988) 431; P. Candelas, P.S. Green, and T. Hübsch, Phys. Rev. Lett.62 (1989) 1956; Nucl. Phys.B330 (1990) 49; P. Candelas and X.C. de la Ossa, Nucl. Phys. B342 (1990) 246.

[18] I. Antoniadis, E. Gava, K. Narain and T. Taylor, ICTP Preprint IC-95-177, hepth/9507115.

[19] E. Witten, Nucl. Phys. B443 (1995) 85 and references therein.

[20] A. Strominger, Nucl. Phys. B451 (1995) 96, hep-th/9504090.

[21] B. Greene, D. Morrison and A. Strominger, Nucl. Phys. B451 (1995) 109, hepth/9504145.

[22] B. Greene, C. Vafa and N. Warner, Nucl. Phys. (1989) 371;

E. Witten, Nucl. Phys. B403 (1993) 159. 
[23] B. Lian and Zuckerman, Phys. Lett. B266 (1991) 21;

S. Mukherji, S. Mukhi and A. Sen, Phys. Lett. B266 (1991) 337;

P. Bouwknegt, J. McCarthy and K. Pilch, Comm. Math. Phys. (1992) 541.

[24] E. Witten, Nucl. Phys. B373 (1992) 187;

E. Witten and B. Zwiebach, Nucl. Phys. B377 (1992) 55.

[25] D. Ghoshal, D. Jatkar and S. Mukhi, Nucl Phys. B395 (1993) 144.

[26] F. Klein, Lectures on the icosahedron and solution of equation of the fifth degree, [English translation (1956)], Dover.

[27] P. Slodowy, in Lecture Notes in Mathematics 1008, Springer (1983).

[28] B. Gato-Rivera and A. Semikhatov, Phys. Lett. B288 (1992) 38;

M. Bershadksy, W. Lerche, D. Nemeschansky and N. Warner, Nucl. Phys. B401 (1993) 304.

[29] D. Ghoshal, P. Lakdawala and S. Mukhi, Mod. Phys. Lett. A8 (1993) 3187.

[30] R. Dijkgraaf, E. Verlinde and H. Verlinde, Nucl. Phys. B352 (1991) 59.

[31] C. Vafa, Mod. Phys. Lett. A6 (1991) 337.

[32] P. Griffiths and J. Harris, Principles of Algebraic Geometry, Wiley-Interscience (1978).

[33] A. Losev, Theor. Math. Phys. 95 (1993) 595.

[34] I. Klebanov and D. Lowe, Nucl. Phys. B363 (1991) 543.

[35] T. Eguchi, H. Kanno, Y. Yamada and S.-K. Yang, Phys. Lett. B298 (1993) 73.

[36] D. Gross and I. Klebanov, Nucl. Phys. B344 (1990) 475.

[37] A. Dhar, G. Mandal and S. Wadia, CERN Preprint CERN-TH-95-186, hep-th/9507121.

[38] E. Witten, IAS Preprint IASSNS-HEP-95-63, hep-th/9507121.

[39] P. Aspinwall and D. Morrison, Phys. Lett. B334 (1994) 79.

[40] M. Bershadsky, V. Sadov and C. Vafa, Harvard preprint HUTP-95/A035, hepth/9510225. 